\title{
Experimental Study on the Effects of Mechanical and Physical Characteristics on Walnut Shucking
}

\author{
Hongmei Xu, Shuiping Yan, and Yapeng Bai \\ College of Engineering, Huazhong Agriculture University, Wuhan 430070, China

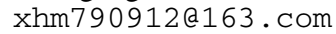

\begin{abstract}
The mechanical and physical characteristics have great impact on the shell shucking of walnut. Taking the shucking force as the evaluation indicator, the effects of mechanical and physical characteristics, which include the loading orientation, loading rate, moisture content and walnut partical size, on walnut shucking were investigated by means of single factor test. The results show that: 1) the loading orientation and loading rate affect the shucking force significantly, and the needed force is the minimum when the walnut is horizontally placed and loaded at the rate of $50 \mathrm{~mm} / \mathrm{min} ; 2$ ) the moisture content has significant effects on the shucking force, and with the increase of drying time, the moisture content of walnut decrease gradually, which results in the increases of the needed shucking force;3) the particle size has no great effect on the shucking force, but great difference in particle size usually corresponds to different walnut shucking force. In order to investigate the shucking technology and design the efficient sheller, it's highly significant to study on the mechanical and physical characteristics of walnut.
\end{abstract}

Keywords: Walnut, Shucking, Mechanical Characteristic, Physical Property.

\section{Introduction}

Walnut is one of the four nuts in the world. In recent years, with the great increase of walnut yield, the walnut foods have developed in the direction of deep processing. However, a critical step for the deep processing of walnut is to shuck the shell and take the meat. Because of the irregular shape and smaller clearance between shell and kernel, it's very difficult for walnut to shuck the shell and take the meat [1].Nowadays, because of the unreasonable force-applying forms, most walnut shellers used in domestic are of larger breaking rate and behave themselves with poor shucking quality $[2,3]$.

Mechanical and physical characteristics can provide fundamental data for developing the sheller and realizing the effective separation of walnut shell and meat. In order to design the highly effective and low damage sheller, mechanical and physical characteristics of walnut must be first investigated.

Taking the dried leatheroid walnut as the research object, the compression test was conducted on the universal material testing machine. The effects of mechanical and physical characteristics, which include the loading orientation, loading rate, moisture content and walnut partical size, on the shucking force of walnut were investigated by 
means of single factor test. The research results are of great significance for improving the working performance of walnut sheller and designing the new and high-efficiency shucking equipments.

\section{Materials and Methods}

\subsection{Materials}

The sold leatheroid walnut from Yunnan was selected as the specimen, and the experiment has been finished in the comprehensive laboratory of College of Engineering, Huazhong Agriculture University, in May 2011.

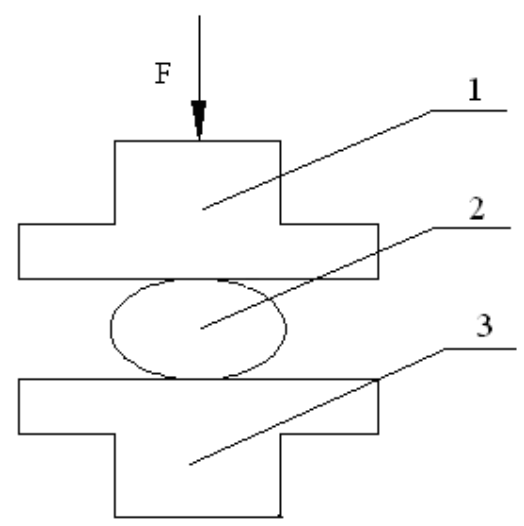

\section{Upper platen 2.Specimen 3. Bottom platen}

Fig. 1. Sketch of the loading device

\subsection{Devices}

The experiment was conducted on the universal material testing machine of RGT2000-10, and the loading rate varies from $0.01 \mathrm{~mm} / \mathrm{min}$ to $500 \mathrm{~mm} / \mathrm{min}$.Fi.1 shows the sketch of the loading device. The pressure imposed on the walnut specimen by the plate-type pressure head is measured by the force transducer.

\subsection{Methods}

The experiment was conducted on the universal material testing machine, and the specimen was applied with the static load. In the beginning of the experiment, put the specimen on the bottom platen of the testing machine, and adjust the location of the upper platen to make sure it contacts with the specimen and imposes a compression force on the specimen. In order to determine the influence of the mechanical and physical characteristics on walnut shucking, the single factor test was 
conducted to investigate the effects of the loading orientation, loading rate, moisture content and particle size on the walnut shucking force. Additionally, for each experimental condition, the experiment was repeated ten times to reduce the test tolerance, and the result is the mean value of 10 tests.

\section{Results and Discussion}

\subsection{Analysis of the Cracking Process of Walnut Shell}

\subsubsection{Cracking Forms of Walnut Shell}

Fig.2 shows the cracking forms of walnut shell when loaded in different orientations. The cracking forms of walnut shell are of two main types, namely, cracking longitudinally and cracking transversely. Because of the structural inhomogeneity and discontinuity in the sewed interface of walnut, it features low compression strength. When the walnut is horizontally placed and applied with a load, the stress concentration and longitudinal crack usually occur. The longitudinal crack propagates in the direction of sewed interface, and plays an important role in the walnut shucking. When the walnut is vertically placed and applied with a load, the cracking form is similar to that of walnut shell when it is horizontally placed. The crack propagates in the direction of sewed interface and behaves itself with definite direction. However, when the walnuts are placed on their sides, as shown in Fig.2 (b), and applied with a load, the transverse crack occurs, and the walnut cracks from the loading point out to its sideways. Furthermore, the extension direction of crack is vertical to the sewed interface, and the crack usually ceases to expand when it reaches the interface [4].

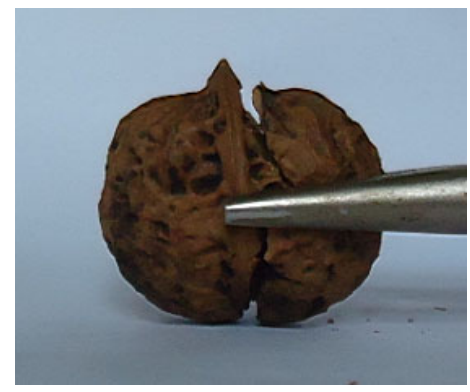

(a) Cracking longitudinally

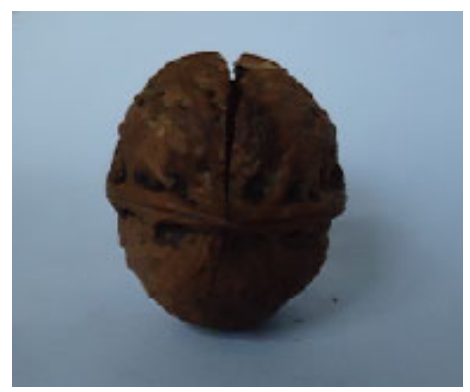

(b) Cracking transversely

Fig. 2. Crack propagation of walnut shell

\subsubsection{Cracking Process of Walnut Shell}

Fig. 3 shows the relation curve between the loading force and deformation of walnut when it is horizontally placed and applied with a load. As shown in the figure, the relation curve deviates from the origin of coordinate system. The reason is that there is a certain clearance between the upper platen and the walnut specimen. Thus, the 
pressure maintains $0 \mathrm{~N}$ until the upper platen contacts with the walnut specimen. The initial deformation is actually the clearance between the upper platen and the walnut specimen.

The cracking process of walnut shell mainly includes three stages, namely, elastic deformation, plastic deformation and sudden rupture. The elastic deformation mainly refers to the early loading stage, in which the pressure linearly increases with the deformation of walnut. Afterwards, with the increase of loading force, the elastic deformation goes hand in hand with plastic deformation. The plastic deformation mainly focuses in the later loading stage. However, when the loading force achieves the peak value for the first time, the walnut begins to crack and the force declines sharply. The peak value mentioned above mainly depends on the compressive strength of walnut, so it's also known as the walnut shucking force. Then, the walnut shell and meat is compressed together, and the required loading force increases again [5] In the course of the experiment, in order to protect the testing machine, the experiment is called for a halt once the walnut begins to crack and the loading force achieves the maximum.

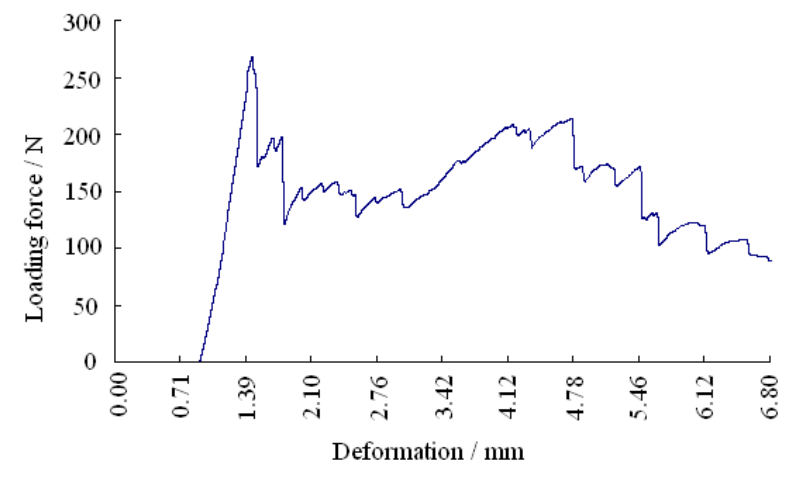

Fig. 3. Relation curve between the loading force and deformation of walnut

\subsection{Analysis of the Influence Factors of Walnut Cracking}

\subsubsection{Effects of Loading Orientation on Walnut Cracking}

Select thirty medium-size walnuts as the specimen, which is coincided with each other in the size and moisture content. Divide the walnuts into three groups, and each group contains ten walnuts. In the course of the experiment, the ten walnuts in group 1 are all horizontally placed as shown in Fig. 4(a).The walnuts in group 2 and group 3 are placed on their sides and vertically placed respectively. Compress the three groups of specimens at the loading rate of $10 \mathrm{~mm} / \mathrm{min}$, and don't cease the experiment until the walnut shell begins to crack, which results in the great reduction of pressure. Record the peak value of the pressure, and regard it as the walnut shucking force. 

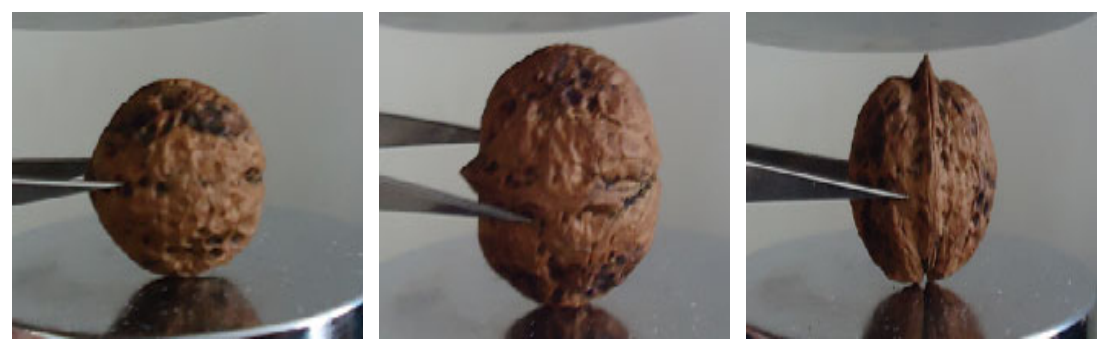

(a) Walnut is horizontally placed (b) Walnut is placed on its side (c) Walnut is vertically placed

Fig. 4. Loading orientation of walnut

Fig.5 shows the testing results of the walnut shucking force when the walnuts are applied with loads in different directions. As shown in the figure, the needed loads are clearly different when the walnuts are applied with loads in different directions. When the walnut is horizontally placed, the needed load is the minimum, which is equal to $195.69 \mathrm{~N}$, and then is the needed load when the walnut is placed on its sides. The needed load is the maximum when the walnut is vertically placed. Variance analysis was conducted for the testing results of walnut shucking force, and the corresponding F-statistic is equal to 12.73 , which is greater than the critical value $F_{\alpha}(2,27)$ $(\alpha=0.05)$. The variance analysis results are consistent with the testing results. It is, therefore, believed that the loading orientation has great impact on the walnut shucking force.

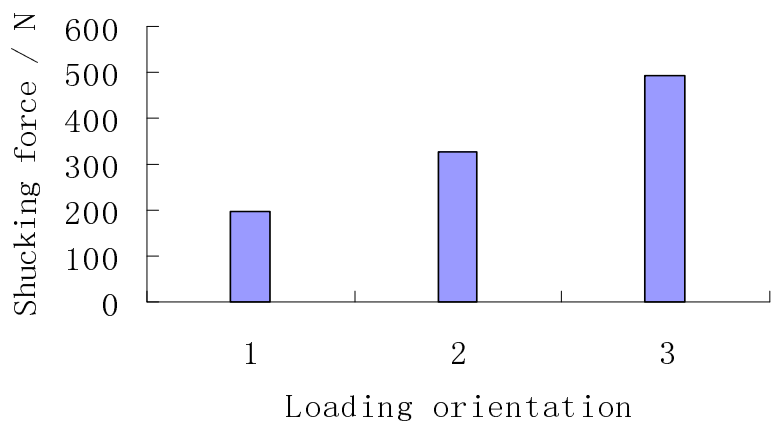

1: Walnut is horizontally placed

2: Walnut is placed on its side

3: Walnut is vertically placed

Fig. 5. Shucking force of walnut with different loading orientation

\subsubsection{Effects of Loading Rate on Walnut Cracking}

Select thirty medium-size walnuts as the specimen, which is coincided with each other in the size and moisture content. Divide the walnuts into three groups, and each group contains ten walnuts. In the course of the experiment, the thirty walnuts are all 
horizontally placed. Compress the three groups of specimens at the loading rate of $10 \mathrm{~mm} / \mathrm{min}, 30 \mathrm{~mm} / \mathrm{min}$ and $50 \mathrm{~mm} / \mathrm{min}$ respectively.

Fig. 6 shows the testing results of the walnut shucking force when the walnuts are compressed at different loading rates. As shown in the figure, the needed shucking forces are different when the walnuts are compressed at different loading rates. When the walnut is compressed at the loading rate of $50 \mathrm{~mm} / \mathrm{min}$, the needed load is the minimum, which is equal to $114.04 \mathrm{~N}$, and then is the loading rate of $10 \mathrm{~mm} / \mathrm{min}$. The needed load is the maximum when the walnut is compressed at the loading rate of $30 \mathrm{~mm} / \mathrm{min}$. Variance analysis was conducted for the testing results of walnut shucking force when the walnuts are compressed at different loading rates, and the corresponding F-statistic is equal to 8.46, which is greater than the critical value $F$ $\alpha(2,27)(\alpha=0.05)$. So it can be concluded that the loading rate affects the walnut shucking force significantly.

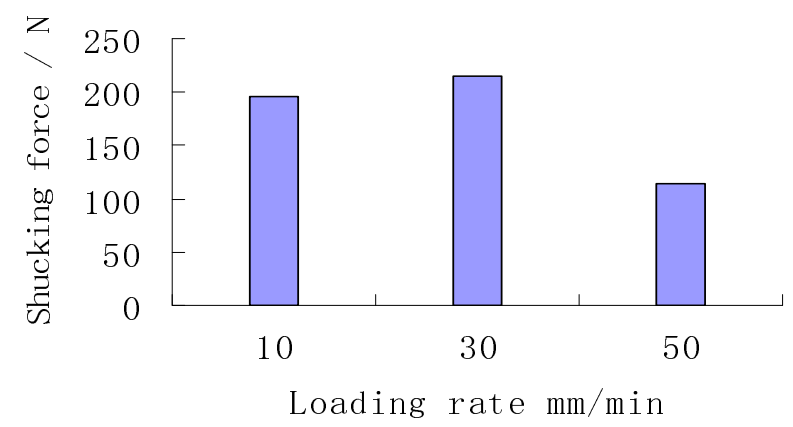

Fig. 6. Shucking force of walnut with different loading rate

\subsubsection{Effects of Particle Size on Walnut Cracking}

Select thirty walnuts as the specimen, which is coincided with each other in the moisture content. Divide the walnuts into three groups, and each group contains ten walnuts. The ten walnuts in group 1 with smaller size ranges from $23 \mathrm{~mm}$ to $27 \mathrm{~mm}$ in height when they are horizontally placed. The ten walnuts in group 2 are of medium size, and range from $28 \mathrm{~mm}$ to $32 \mathrm{~mm}$ in height when they are horizontally placed. The ten walnuts in group 3 are the biggest ones, and range from $33 \mathrm{~mm}$ to $37 \mathrm{~mm}$ in height when they are horizontally placed. In the course of the experiment, the thirty walnuts are all horizontally placed, and compressed at the loading rate of $10 \mathrm{~mm} / \mathrm{min}$.

Fig.7 shows the testing results of the shucking force of walnut with different particle size. For the walnuts with different particle size, the needed loads are different, but the difference is not large. The needed shucking force of the mediumsize walnuts is the minimum, which is equal to $166.47 \mathrm{~N}$, and then is the smaller ones. The needed shucking force of the large-size walnuts is the maximum. Variance analysis was conducted for the testing results of the shucking force of walnut with different particle size, and the corresponding F-statistic is equal to 1.43 , which is less than the critical value $F_{\alpha}(2,27)(\alpha=0.05)$.It can be concluded that particle size has no great effect on the walnut shucking force. 


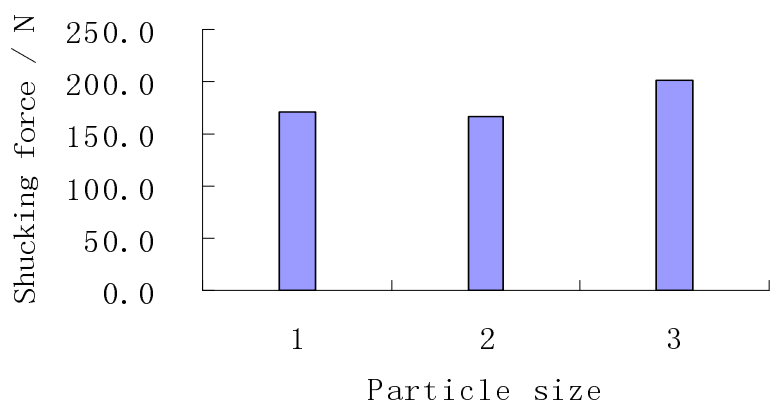

1: Walnut is of larger size

2: Walnut is of medium size

3: Walnut is of smaller size

Fig. 7. Shucking force of walnut with different particle size

\subsubsection{Effects of Moisture Content on Walnut Cracking}

Select thirty medium-size walnuts as the specimen, which is coincided with each other in the particle size .Divide the walnuts into three groups, and each group contains ten walnuts. In order to investigate the effect of the moisture content on the walnut shucking force, the walnuts must be pretreated before the compression test. The drying experiment is one of the most important preprocessing methods, which is usually used to control the moisture content of the samples. In this research, the drying experiments were conducted for three groups of walnut specimens, and the corresponding drying time is $0 \mathrm{~h}, 1 \mathrm{~h}$ and $2 \mathrm{~h}$ respectively. Afterwards, the walnuts are all horizontally placed, and compressed at the loading rate of $10 \mathrm{~mm} / \mathrm{min}$.

Fig.8 shows the testing results of the shucking force of walnut with different drying time and moisture content. As shown in the figure, different drying time corresponds to different walnut shucking force. When the drying time is $0 \mathrm{~h}$, the needed shucking force is the minimum, which is equal to $114.49 \mathrm{~N}$, and then is the walnuts with the drying time of $1 \mathrm{~h}$. The needed shucking force of the walnuts with the drying time of

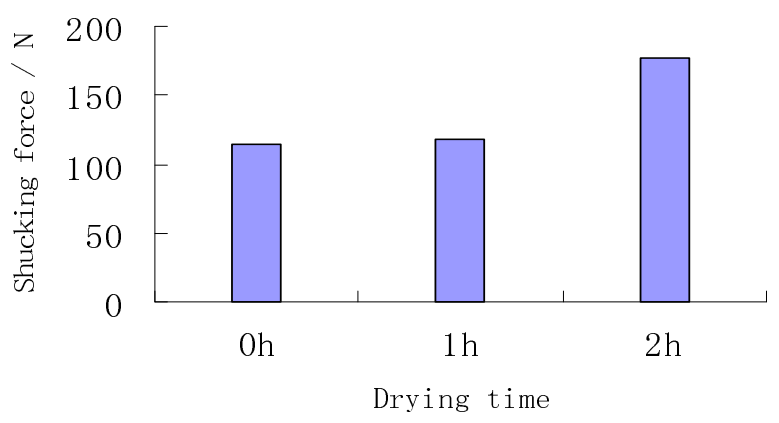

Fig. 8. Shucking force of walnut with different moisture content 
$2 \mathrm{~h}$ is the maximum. It can be seen that the moisture content of walnuts decreases with the increase of drying time. As a result, the needed shucking force increases which makes walnut shucking more difficult. Variance analysis was conducted for the testing results of the shucking force of walnuts with different moisture content, and the corresponding F-statistic is equal to 49.23, which is greater than the critical value $F_{\alpha}(2,27)(\alpha=0.05)$. Therefore, it can be concluded that the drying time and moisture content has great effects on the walnut shucking force.

\section{Conclusions}

Take the shucking force as the evaluation indicator, and the effects of the loading orientation, loading rate, moisture content and walnut particle size on walnut shucking were investigated by means of single factor test. The conclusions can be described as follows:

(1) Loading orientation has great impact on the walnut shucking force. When the walnuts are horizontally placed, the needed shucking force is the minimum, and then is the walnuts placed on their side. The needed shucking force of the walnuts vertically placed is the maximum.

(2) Loading rate affects the walnut shucking force significantly. When the walnut is compressed at the loading rate of $50 \mathrm{~mm} / \mathrm{min}$, the needed shucking force is the minimum, and then is the loading rate of $10 \mathrm{~mm} / \mathrm{min}$. The needed force is the maximum when the walnuts are compressed at the loading rate of $30 \mathrm{~mm} / \mathrm{min}$.

(3) Moisture content has significant effects on the shucking force, and with the extension of drying time, the moisture content of walnut decrease gradually, which results in the increases of the needed shucking force.

(4) Particle size has no great effect on the shucking force, but great difference in particle size usually corresponds to different walnut shucking force.

(5) The cracking forms of walnut shell are of two main types, namely, cracking longitudinally and cracking transversely. When the walnuts are horizontally and vertically placed and applied with a load, the longitudinal crack usually occurs. The transverse crack occurs only when the walnuts are placed on their sides.

Acknowledgement. This research was supported by Student's Science \& Technology Innovation Fund of Huazhong Agricultural University "Research on the prediction of the acoustic performance for vehicle-use sound packages based on FE-SEA hybrid model" under grant Nos. 11028.

\section{References}

1. Jianxin, S., Haijun, Z., Dongjun, X.: Technology for breaking walnut shell based on finite element analysis. Transactions of the CSAE 21(3), 185-188 (2005)

2. Yuande, D., Jianxin, S., Yuanyuan, Q.: Effect of the shell-breaking and kernel-taking about walnut for various shell-breaking methods. Academic Periodical of Farm Products Processing 9, 4-9 (2007) 
3. Jianxin, S., Dongjun, X.: Discussions on the status and problems of the walnut shellers at home and abroad. Agriculture machinery of Xinjiang 6, 29-32 (2001)

4. Yichuan, H., Jianxin, S.: Analysis and Experiment on Mechanical Characteristic of Walnut Shell. Journal of Xinjiang Agricultural University 32(6), 70-75 (2009)

5. Lihua, Z., Wen, Z., Wen, Q., Mingxia, L.: Mechanical Characteristics of Peanut Cracking. Food Science 31(13), 52-55 (2010) 